\title{
Efeitos da Fotobiomodulação em Células Tumorais in vitro: Revisão da Literatura
}

\author{
Photobiomodulation Effects on Tumor Cells in Vitro: Literature Revision
}

\author{
Jéssica Lucio da Silva*a; Ana Flávia Spadaccini Silva-de-Oliveira; ${ }^{\text {a }}$ Danielle Gregorio ${ }^{a}$; Rodrigo Antonio Carvalho \\ Andraus $;$ Luciana Prado Maia ${ }^{\text {ab }}$
}

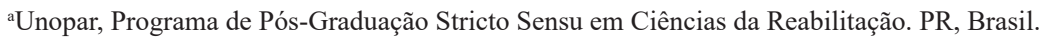

bUnopar, Programa de Pós-Graduação Stricto Sensu em Odontologia PR, Brasil.

*Email: jessicaldsilva@hotmail.com

\begin{abstract}
Resumo
Atualmente, novos métodos de tratamento contra o câncer estão sendo investigados para a obtenção de um bom prognóstico e menor índice de efeitos colaterais, e uma das terapias atuais sob investigação é a Fotobiomodulação (FBM). Sabe-se que a proliferação de células normais e tumorais pode ser estimulada ou inibida pela FBM, e esse processo vai depender dos parâmetros utilizados. O objetivo deste estudo foi conduzir uma revisão da literatura, a fim de avaliar os efeitos biológicos de diferentes parâmetros da FBM sobre cultura de células tumorais (osteossarcoma). Realizou-se busca na literatura através das bases de dados PubMed/MEDLINE, Cochrane Library e EMBASE. Os estudos mostraram que a FBM pode ser utilizada em células cancerígenas de forma a diminuir a sua proliferação, contudo, a falta de padronização dos protocolos de irradiação a laser para investigações in vitro não permite estabelecer os parâmetros ideais para esse fim. Com base nos achados relatados se pode sugerir que a FBM, em parâmetros específicos, pode promover a bioestimulação ou até mesmo a inibição das células cancerígenas, sendo necessários mais estudos para determinar os protocolos de tratamento ideais e, por isso, deve ser usada com cautela na prática clínica.
\end{abstract}

Palavras-chave: Terapia com Luz de Baixa Intensidade. Terapia a Laser. Osteossarcoma.

\begin{abstract}
Currently new treatment methods against cancer have been investigated to obtain a good prognosis and a lower rate of side effects, and one of the current therapies under investigation is Photobiomodulation (PBM). It is known that the normal and tumor cells proliferation can be stimulated or inhibited by PBM, and this process will depend on the parameters used. The objective of this study was to conduct a literature review in order to assess the biological effects of different PBM parameters on tumor cell culture (osteosarcoma). Literature search was performed through the PubMed/MEDLINE, Cochrane Library and EMBASE databases. Inclusion criteria were: to be an in vitro study with cancer cells, and to have at least one treatment group with PBM and a control group (without treatment). Studies have shown that PBM can be used in cancerous lesions in order to decrease the proliferation of these cells, however, the lack of standardization of laser irradiation protocols for in vitro investigations does not allow to establish the ideal parameters for this purpose. Based on the reported findings, it can be suggested that PBM in specific parameters may promote biostimulation or even inhibition of cancer cells. More studies are necessary to determine the ideal treatment protocols, and, because of this, it should be used with caution in clinical practice.
\end{abstract}

Keywords: Low Intensity Light Therapy. Laser therapy. Osteosarcoma.

\section{Introdução}

O câncer é uma doença caracterizada pela proliferação descontrolada de um grupo de células, mais rápido que as células normais circundantes. Atualmente, o câncer é um problema de saúde sério, em função da mortalidade e morbidade e a um aumento na frequência em muitas partes do Mundo (DEVITA; ELlMAN; ROSENBERG, 2008). Segundo o Instituto Nacional de Câncer (Inca), o Brasil poderá ter 625 mil novos casos de câncer em 2020, entre esses, 50,3\% deverão ocorrer em homens e 49,7\% em mulheres (INCA, 2019).

O tratamento dos diferentes tipos de câncer consiste em cirurgia associada à quimioterapia e/ou radioterapia. Contudo, é importante a procura de uma melhor abordagem terapêutica para o câncer, visando minimizar os efeitos adversos, diminuir a incidência das metástases, aumentar o tempo de sobrevida e proporcionar melhor qualidade de vida aos pacientes (ANDRADE; CLARCK; FERREIRA, 2014).-

A Fotobiomodulação (FBM) utilizando Terapia Laser de Baixa Potencia (TLBP) vem sendo amplamente aplicada em diversas áreas da saúde, com base em seus efeitos bioestimuladores e biomoduladores, o que significa que a irradiação em parâmetros específicos é capaz de alterar o comportamento celular (KARA; ORBACK, 2009). O mecanismo da FBM é baseado diretamente na aplicação de energia luminosa bioestimulatória às células. Os fotorreceptores celulares absorvem a luz, e podem transferila para as mitocôndrias para produzir ATP (MURAYAMA, et al..2012).

Com o aumento da vasodilatação via síntese de ATP, o uso de oxigênio é aumentado e a atividade das enzimas citoplasmáticas com os ácidos nucléicos estimula a mitose celular (KARA, et al.,2018). 
O potencial proliferativo aumenta não só a angiogênese, mas também a síntese de fatores de crescimento, de metabólitos inflamatórios e, também, modula células imunológicas (FRIGO, et al., 2009).

Sendo assim, a FBM pode ativar células pré-cancerígenas ou aumentar tecidos cancerígenos existentes em caso de situações clínicas não detectadas (BAMPS; DOK; NUYTS, 2018), e isso faz com que se evite o uso da FBM como modelo terapêutico em pacientes com câncer.

No entanto, evidências científicas sobre a FBM vêm apontando uma melhor compreensão quanto aos seus efeitos no processo de reparo do tecido ósseo danificado e seu lugar na oncologia (BASSO et al.,2014), já que esses efeitos dependem de parâmetros específicos, como o comprimento de onda, densidade de energia e tempo de aplicação no tecido biológico (BARASCH et al.,2016; CROUS; ABRAHHAMSE, 2016; SCHALCHH et al.,2019). Dependendo dos parâmetros utilizados, por exemplo, com doses elevadas, a FBM pode resultar em uma terapia efetiva para bioinibição, não apenas reduzindo a proliferação celular, mas também induzindo a apoptose de células tumorais (DA SILVA et al., 2019).

Contudo, não existe um consenso clínico para aplicação da FBM em regiões com a presença de tumores malignos, já que a literatura apresenta resultados distintos ao utilizá-la em doses elevadas, demonstrando tanto resultados satisfatórios (BASHARDOUST et al., 2010; RENNO, et al., 2010) quanto à inibição do reparo tecidual, resultando em efeitos agressivos em nível celular (KARA et al., 2010).

Considerando os efeitos benéficos do laser como terapia para diferentes patologias, a possibilidade de utilização do mesmo em pacientes com câncer seria de grande interesse para área clínica. No entanto, para isso, devem-se levar em consideração os efeitos dessa terapia em nível celular, de acordo com os parâmetros de irradiação aplicados. Portanto, o objetivo do presente estudo foi avaliar, por meio de uma revisão de literatura, os efeitos biológicos de diferentes parâmetros da FBM sobre cultura de células osteossarcoma.

\section{Desenvolvimento}

\subsection{Seleção dos estudos}

Para a elaboração do presente estudo foi realizada uma busca eletrônica de artigos científicos publicados em periódicos nacionais e internacionais, nas bases de dados PubMed/ MEDLINE, Cochrane Library e EMBASE, utilizando a seguinte estratégia de busca: ("laser therapy"[MeSH Terms] OR ("laser"[All Fields] AND "therapy"[All Fields]) OR "laser therapy”[All Fields]) AND ("Cancer Cell”"[Journal] OR ("cancer"[All Fields] AND "cell"[All Fields]) OR "cancer cell"'[All Fields]).

\subsection{Fotobiomodulação}

O termo Laser - Light Amplification By Stimulated Emission of Radiation (amplificação de luz por emissão estimulada de radiação) (DAVIDOVICH,2015) foi descrito, inicialmente, por Albert Einstein, em 1917, de forma teórica, mas apenas em 1960, Theodore H. Maiman anunciou o seu funcionamento com fins terapêuticos (BAYAT et al., 2018), sendo aplicado clinicamente para a cicatrização de feridas, alívio da dor, inflamação e várias condições ortopédicas (HAMBLIN; NELSON; STRAHAN, 2018).

Atualmente, o laser de baixa potência tem ganhado grande destaque mundial nas Ciências da Saúde em função de busca por formas menos invasivas de tratamento. Com isso, tornouse um método muito utilizado na maioria dos países e é estudada mundialmente, e a sua popularidade está relacionada não só a sua ação menos invasiva, mas também por ser atérmica, indolor, baixo custo (DE OLIVEIRA et al., 2012) e com tempo de aplicabilidade inferior aos demais recursos fisioterapêuticos (LINS et al., 2010). Grande variedade de condições patológicas utilizando a FBM é descrita na literatura, tais como: a modulação de processo inflamatório (CAETANO; ZANUTO, 2013), aceleração do reparo tecidual (LORETI, et al., 2015), alívio de dor e tratamento de algumas desordens neurológicas (STEIN et al., 2018).

Vários estudos in vitro e in vivo mostram que a FBM está correlacionada com a cicatrização acelerada de feridas em função da estimulação de processos celulares, como migração e diferenciação celular (ALGHAMDI; KUMAR; MOUSSA, 2012; DJAVID et al.,2018). Sabe-se que a cadeia respiratória nas mitocôndrias é estimulada pela FBM, o que resulta em um aumento na produção de adenosina trifosfato (ATP) e, portanto, no aumento da síntese de Ácido Desoxirribonucleico (DNA), Ácido Ribonucleico (RNA) e proteínas (GOMES HENRIQUES et al., 2014). Além disso, a FBM aumenta a proliferação celular, levando ao risco indesejado de estimular a proliferação de células cancerígenas.

Contudo, a efetividade da FBM e seus respectivos efeitos no tecido exposto à irradiação se encontram dependentes dos parâmetros específicos aplicados, tais como: comprimento de onda $(\lambda)$, densidade de potência, densidade de energia (dE) e tempo de tratamento (BENSADOUN; NAIR, 2012), pois dependendo dos parâmetros utilizados, as células ou tecidos são estimulados tanto a um efeito bioestimulatório quanto bioinibitório (ANDRADE et al., 2014; CROUS; ABRAHAMSE, 2016).

Levando em consideração a sua eficácia e sua ação não invasiva, a FBM vem sendo estudada como uma opção de tratamento para o câncer (BAXTER et al., 2017).

\subsection{Ação da Fotobiomodulação em células tumorais}

Os efeitos da FBM na proliferação e diferenciação celular foram investigados in vitro usando linhas celulares malignas, e geraram dados conflitantes em diferentes tipos de células tumorais e parâmetros aplicados no laser (PINHEIRO et al., 2002). A ação dos lasers na atividade proliferativa é um tópico controverso, porque a ação é encontrada em estudos de 
culturas celulares tanto inibindo, quanto proliferando ou até mesmo sendo indiferentes quanto ao crescimento (DA SILVA et al., 2019). Além disso, existem algumas especulações que o laser poderia aumentar o crescimento do tumor em doenças neoplásicas (FRIGO et al., 2009).

Werneck et al. (2005) descreveram que a célula tumoral possui deficiência nutricional, em decorrência de sua intensa atividade metabólica e, por isso, é susceptível a ação da FBM. Porém, estudos in vitro mostram que os parâmetros utilizados no laser para aplicação da luz e o tipo celular podem modular as respostas obtidas, como a resposta citotóxica (RAMOS SILVA et al., 2016) e o potencial proliferativo celular (FRIGO et al., 2009).

Dependendo dos parâmetros do laser, o mesmo pode estimular o processo proliferativo tanto em células normais quanto em células tumorais. O uso da FBM durante o processo neoplásico pode aumentar a proliferação e diferenciação celular, já que a mesma apresenta efeitos biomoduladores significantes, e isso não é um efeito desejável em neoplasias (RENNO et al., 2010).

Objetivando elucidar o mecanismo biológico da proliferação celular, Sroka et al, (1999) avaliaram o efeito da FBM em células de diferentes origens e diferentes graus de malignidade, comparando-as com células normais. Foi verificado um aumento do padrão mitótico em células benignas e malignas, após irradiação com doses no intervalo de 4 a $8 \mathrm{~J} / \mathrm{cm}^{2}$. No entanto, se observou uma redução na taxa de proliferação celular quando a dose ultrapassava esse intervalo de energia, independente do comprimento de onda.

Pinheiro et al. (2002) realizaram um estudo analisando o efeito da FBM em células malignas in vitro. Em particular, eles estudaram células cancerígenas do tipo epitelial, expondo-as a irradiação nos comprimentos de onda de 635 e $670 \mathrm{~nm}$. As células irradiadas, em ambos os comprimentos de onda, proliferaram mais do que o grupo controle não irradiado. $\mathrm{O}$ grupo exposto a $670 \mathrm{~nm}$ proliferou mais do que o grupo a $635 \mathrm{~nm}$. Os autores concluem que a exposição à luz do laser poderia aumentar significativamente, a proliferação de células cancerígenas.

Carnevalli et al. (2003) demonstraram em seu estudo que células CHO K-1 cultivadas e irradiadas com laser de baixa intensidade $\left(830 \mathrm{~nm}\right.$ e $\left.2 \mathrm{~J} / \mathrm{cm}^{2}\right)$ exibiram maior síntese de ATP e capacidade mitótica quando submetidas ao estresse nutricional, quando comparadas com células não-irradiadas do grupo controle.

Renno et al. (2007) investigaram os efeitos da irradiação laser de 670, 780 e $830 \mathrm{~nm}$ na proliferação de células provenientes do osteossarcoma humano e verificaram que apenas a dose de $10 \mathrm{~J} / \mathrm{cm}^{2}$ a $830 \mathrm{~nm}$ foi capaz de aumentar a proliferação de osteoblastos, enquanto as densidades de energia de 1, 5 e $10 \mathrm{~J} / \mathrm{cm}^{2}$ a $780 \mathrm{~nm}$ diminuíram a proliferação. As células de osteossarcoma não foram afetadas pela irradiação com laser a $830 \mathrm{~nm}$, enquanto o laser a $670 \mathrm{~nm}$ teve um efeito proliferativo leve.
Frigo et al. (2009) utilizaram células de melanoma murinho (B16F10) para irradiação com o laser emitindo um comprimento de onda de $660 \mathrm{~nm}$, potência de $50 \mathrm{~mW}$ e irradiância de $2,5 \mathrm{~W} / \mathrm{cm}^{2}$ nas densidades de energia de $150 \mathrm{~J} /$ $\mathrm{cm}^{2}$ e $1050 \mathrm{~J} / \mathrm{cm}^{2}$ fracionando a energia entregue em três dias consecutivos a $9 \mathrm{~J}$ e $63 \mathrm{~J}$ respectivamente. Após realizarem o teste de exclusão com azul de tripan, não identificaram diferença estatística significativa em relação ao grupo controle.

Por outro lado, Basso et al. (2014) detectaram que a FBM no comprimento de onda de $780 \mathrm{~nm}$ e a uma densidade de energia de $0,5 \mathrm{~J} / \mathrm{cm}^{2}$ promoveu aumento na proliferação de células osteoblásticas de osteossarcoma (Saos-2), enquanto que as doses de 3 e $7 \mathrm{~J} / \mathrm{cm}^{2}$ reduziram a atividade da fosfatase alcalina.

Um estudo realizado por Liang et al. (2015), utilizando células epiteliais originadas de adenocarcinomas (ASTC-a-1 de adenocarcinoma de pulmão humano), mostrou que o comprimento de onda de $632,8 \mathrm{~nm}$ e a densidade de energia variando de 3 a $15 \mathrm{~J} / \mathrm{cm}^{2}$ aumentam, significativamente, o crescimento celular, enquanto que a densidade de energia de $50 \mathrm{~J} / \mathrm{cm}^{2}$ inibe a viabilidade celular. Porquanto, Liang et al. (2015) demonstraram que a aplicação de altas densidades de energia $\left(60 \mathrm{~J} / \mathrm{cm}^{2}\right)$ induz a apoptose em células cancerígenas.

Uma revisão sistemática publicada recentemente, por Da Silva et al. (2019), sugeriu que a FBM pode ser utilizada em lesões cancerígenas, a fim de diminuir a proliferação das células cancerígenas, dependendo dos parâmetros utilizados; no entanto, a falta de padronização dos protocolos de irradiação a laser para investigações não permite o estabelecimento de parâmetros ideais para esse fim, portanto, a FBM deve ser usado com cautela em pacientes com câncer até que mais estudos sejam realizados.

Dessa forma, torna-se importante a procura de uma melhor abordagem terapêutica para o câncer, levando em consideração a necessidade de minimizar os efeitos adversos, diminuir a incidência das metástases, aumentar o tempo de sobrevida e proporcionar melhor qualidade de vida aos pacientes, tendo em vista que dependendo da densidade de energia aplicada nessas células, a FBM pode estimular a metástase, sendo uma forma de terapia contraindicada, pois irá acarretar no aumento da doença, ou poderá inibir a proliferação celular, sendo considerada uma terapia favorável, que pode ser utilizada como coadjuvante no tratamento de pacientes oncológicos.

Resultados experimentais com cultura de células não são facilmente comparáveis por depender de diversos fatores: parâmetros de irradiação, diferentes manejos com as células, diferentes técnicas de laboratório e, ainda, a irradiação com diferentes intervalos de tempo (WERNECK et al., 2005). Dessa forma, a falta de padronização das condições experimentais e as múltiplas combinações dos parâmetros da FBM explicam os resultados divergentes, que são obtidos quando se irradiam células tanto benignas quanto malignas com o laser terapêutico.

Outra consideração importante é que o sucesso dos 
resultados in vitro nem sempre reproduz diretamente resultados positivos em aplicações in vivo, já que outras variáveis estão presentes neste último modelo (HENRIQUES; CAZAL; DE CASTRO, 2010).

Essa revisão enfatiza a necessidade de mais estudos se utilizando várias combinações de comprimentos de onda e doses em diferentes linhagens de células in vitro e in vivo, com a finalidade de padronizar os desenhos experimentais, tornando possível uma comparação dos resultados e, consequentemente, determinando os parâmetros da FBM que deverão ser utilizados para obtenção dos efeitos desejados.

\section{Conclusão}

Com base na análise dos dados obtidos, no presente estudo, pode-se sugerir que a FBM em parâmetros específicos pode promover a bioestimulação ou até mesmo a inibição das células cancerígenas, devendo ser usada com cautela na prática clínica, tendo em vista o risco de aumentar o tecido canceroso existente. No entanto, ao considerar o uso da terapia como um tratamento bioinibitório, nesse tipo celular, são necessários mais estudos para elucidar os principais fatores responsáveis pelos diferentes comportamentos, nas células tumorais, em resposta à luz laser, para determinar quais parâmetros levariam a tais efeitos.

\section{Referências}

AL-ROMAIH, K. et al. Chromosomal instability in osteosarcoma and its association with centrosome abnormalities. Cancer Genetics Cytogenetics, v.144, n.2, p.91-99, 2003.

ALGHAMDI, K.M.; KUMAR, A.; MOUSSAN. A. Low-level laser therapy: a useful technique for enhancing the proliferation of various cultured cells. Lasers Med. Scie., v.27, n.1, p.237-249, 2012.

ANDRADE, F. S. S. D.; CLARK, R. M. O.; FERREIRA, M. L. Efeitos da laserterapia de baixa potência na cicatrização de feridas cutâneas. Rev. Col. Bras Cirurgiões, v.41, n.2, p.129-133, 2014.

BAMPS, M.; DOK, R.; NUYTS, S. Low-level laser therapy stimulates proliferation in head and neck squamous cell carcinoma cells. Frontiers in Oncology, v.8, p.1-6, 2018

BARASCH, A. et al. Effects of pre-radiation exposure to LLLT of normal and malignant cells. Supportive Care in Cancer, v.24, n.6, p.2497-2501, 2016.

TAJALI, S. et al. Effects of low power laser irradiation on bone healing in animals: a meta-analysis. J. Orthop. Surg. Res., v.5, n.1, p.1-10, 2010.

BASSO, F. G. et al. Low-level laser therapy for osteonecrotic lesions: effects on osteoblasts treated with zoledronic acid. Supportive Care in Cancer, v.22, n.10, p.2741-2748, 2014.

BAXTER, G.D. et al.Low levellaser therapy(Photobiomodulation therapy) for breast cancer-related lymphedema: a systematic review. BMC Cancer, v.17, n.1, p.833-846, 2017

BAYAT, M. et al. Comparison of the in vitro effects of low-level laser therapy and low-intensity pulsed ultrasound therapy on bony cells and stem cells. Prog. Biophys. Mol. Biol., v.133, p.3648, 2018.

BENSADOUN, R. J.; NAIR, R. G. Low-level laser therapy in the prevention and treatment of cancer therapy-induced mucositis:
2012 state of the art based on literature review and meta-analysis. Current Opinion Oncol., v.24, n.4, p.363-370, 2012.

CAETANO, H.R.S.; ZANUTO, É.A.C. Ação da laserterapia de baixa intensidade sobre parâmetros bioquímicos. uma revisão de literatura. Colloquium Vitae, v.5, p.63-69, 2013.

CARNEVALLI, C. M. M. et al. Laser light prevents apoptosis on CHO K-1 cell line. J. Clin. Laser Med. Surg., v.21, n.4, p.193196, 2003.

CHEN, X.; ZHANG, Y. Bmp-2 and mir-29c in osteosarcoma tissues on proliferation and invasion of osteosarcoma cells. Oncology Letters, v.17, n.6, p. 5389-5394, 2019.

CROUS, A.; ABRAHAMSE, H. Low-Intensity Laser Irradiation at $636 \mathrm{~nm}$ Induces Increased Viability and Proliferation in Isolated Lung Cancer Stem Cells. Photomed. Laser Surg., v.34, n.11, p.525-532, 2016.

DA SILVA, J. L. et al. Effects of low level laser therapy in cancer cells: systematic review of the literature. Lasers Med. Scie., v.35, p.523-529, 2019.

DAVIDOVICH, L. Os quanta de luz e a ótica quântica. Rev. Bras. Ens. Fís., v.37, n.4, p.4205-4212, 2015.

DEVITA, V.T.; HELlMAN, S., ROSENBERG, A.S. Cancer: principles and practice of oncology. Philadelphia: LippincottRaven Publishers, v.77, n.11, p. 2058, 1997.

DE OLIVEIRA, P. et al. Comparação dos efeitos do laser de baixa potência e do ULTRASSOM de baixa intensidade associado ao Biosilicato ${ }^{\circledR}$ no processo de reparo ósseo em tíbias de ratos. Rev. Bras. Ortop., v.47, n.1, p.102-107. 2012.

DECLERCQ, H. et al. Isolation, proliferation and differentiation of osteoblastic cells to study cell/biomaterial interactions: comparison of different isolation techniques and source. Biomaterial,, v.25, n.5, p.757-768, 2004.

DJAVID, G. E. et al. Photobiomodulation leads to enhanced radiosensitivity through induction of apoptosis and autophagy in human cervical cancer cells. J. Biophotonics, v.10, n.12, p.17321742, 2018.

DU, L. et al. Establishment and characterization of a new highly metastatic human osteosarcoma cell line derived from Saos2. Int. J. Clin. Exp. Pathol., v.7, n.6, p.2871-2882, 2014.

FOGH, J.; FOGH, J. M.; ORFEO, T. One hundred and twenty seven cultured human tumor cell lines producing tumors in nude mice. J.Nat. Cancer Inst., v.59, n.1, p.221-226, 1977.

FRIGO, L. et al. The effect of low-level laser irradiation (InGa-Al-AsP - $660 \mathrm{~nm}$ ) on melanoma in vitro and in vivo. BMC Cancer., v.9, p.1-8, 2009.

GOMES HENRIQUES, Á.C. et al. Low-level laser therapy promotes proliferation and invasion of oral squamous cell carcinoma cells. Lasers Med. Scie., v.29, n.4, p.1385-1395, 2014.

HAMBLIN, M. R.; NELSON, S. T.; STRAHAN, J. R. Photobiomodulation and cancer: what is the truth? Photomed. Laser Surg., v.36, n.5, p.241-245, 2018.

HENRIQUES, Á. C. G.; CAZAL, C.; DE CASTRO, J. F. L. Ação da laserterapia no processo de proliferação e diferenciação: Revisão da Literatura. Rev. Colégio Bras. Cirur., v.37, n.4, p.295302, 2010.

INSTITUTO NACIONAL DE CÂNCER JOSÉ ALENCAR GOMES DA SILVA. Tipos de câncer. Rio de Janeiro: INCA, 2019.

KARA, C. et al.. Low level laser therapy induces increased viability and proliferation in isolated cancer cells. Cell 
Proliferation, v.51, n.2, p.1-6, 2018.

KARA, C.; ORBAK, R. Comparative evaluation of Nd:YAG laser and fluoride varnish for the treatment of dentinal hypersensitivity. J. Endod., v.35, p.971-974, 2009.

LIANG, W. Z. et al. Selective cytotoxic effects of low-power laser irradiation on human oral cancer cells. Lasers in Surg. Med., v.47, n.9, p.756-764, 2015.

LINS, R. D. A. U. et al. Biostimulation effects of low-power laser in the repair process. Anais Bras. Dermatol., v.85, n.6, p.849-855, 2010.

LORETI, E. H. et al. Use of laser therapy in the healing process: a literature review. Photom. Laser Surgery, v.33, n.2, p.104-116, 2015.

MA, J.; GAO, W.; GAO, J. sCLU as prognostic biomarker and therapeutic target in osteosarcoma. Bioengineered, v.10, n.1, p.229-239, 2019.

MIRABELLO, L. et al. Germline TP53 Variants and Susceptibility to Osteosarcoma. J. National Cancer Institute., v.10, n.7, p.5-8, 2015 .

MURAYAMA, H. et al. Low-power 808-nm laser irradiation inhibits cell proliferation of a human-derived glioblastoma cell line in vitro. Lasers Med. Scie., v.27, n.1, p.87-93, 2012.

PAUTKE, C. et al. Characterization of osteosarcoma cell lines mg-63, saos-2 and u-2 os in comparison to human osteoblasts. Anticancer Res,.., v.24, p.3743-3748, 2004.

PINHEIRO, A. L. B. et al. Effects of low-level laser therapy on malignant cells: in vitro study. J. Clin. Laser Med. Surg., v.20, n.1, p.23-26, 2002.
RAMOS SILVA, C. et al. Exploring the effects of low-level laser therapy on fibroblasts and tumor cells following gamma radiation exposure. J. Biophotonics, v.9, n.11-12, p.1157-1166, 2016.

RENNO, A. C. M. et al. Effect of $830 \mathrm{~nm}$ Laser Phototherapy on Osteoblasts Grown In Vitro on Biosilicate ${ }^{\circledR}$ Scaffolds. Photomed. Laser Surg., v.28, n.1, p.131-133, 2010.

RENNO, A. C. et al. The effects of laser irradiation on osteoblast and osteosarcoma cell proliferation and differentiation in vitro. Photomed. Laser Surg., v.25, n.4, p.275-280, 2007.

SCHALCH, T. D. et al. Photobiomodulation is associated with a decrease in cell viability and migration in oral squamous cell carcinoma. Lasers Med. Scie., v.34, n.3, p.629-636, 2019.

SROKA, R. et al. Effects on the mitosis of normal and tumor cells induced by light treatment of different wavelengths. Lasers Med. Scie., v.25, n.3, p.263-271, 1999.

STEIN, C. et al. Acute effects of low-level laser therapy on patients' functional capacity in the postoperative period of coronary artery bypass graft surgery: a randomized, crossover, placebo-controlled trial. Photomed. Laser Surg., v.36, n.3, p.122129, 2018.

WERNECK, C.E. et al. Laser light is capable of inducing proliferation of carcinoma cells in culture: A spectroscopic in vitro study. Photomed. Laser Surg., v.23, n.3, p.300-303, 2005.

YU, Y. et al. Differential expression of osteogenic factors associated with osteoinductivity of human osteosarcoma cell lines. J. Biomed. Mat. Res, Part A, v.70, n.1, p.122-128, 2004. 